\title{
SKILLED HEALTH PERSONNEL ATTENDED DELIVERY AS A PROXY INDICATOR FOR MATERNAL AND PERINATAL MORTALITY: A SYSTEMATIC REVIEW
}

\author{
Yifru Berhan ${ }^{1}$, Asres Berhan²
}

\begin{abstract}
BACKGROUND: Several demographic and health surveys in Africa have shown the high prevalence of home delivery, but little is known how strongly skilled person unattended deliveries are associated with maternal and perinatal mortality. The aim of this review was to assess the gross correlation of maternal mortality ratios (MMR) and perinatal mortality rates (PMR) with the proportion of skilled health personnel attended deliveries

METHODS: In this study, a systematic review was conducted after a computer based literature search was run in the electronic databases from 1990 through September 2013. Bivariate linear regression analyses were done for the proportion of skilled person attended deliveries in relation with MMR, stillbirth and neonatal mortality rates using national survey data of 41 African countries.

RESULTS: African countries with relatively small population sizes and with middle to high income were found to have above $90 \%$ skilled person attended deliveries. Several African countries with a high proportion of skilled person attended deliveries (60\%-100\%) were able to reduce the MMR to the range of 56-370/100,000 live births. Several Sub Saharan African (SSA) countries were far from their northern counterparts. The regression analyses demonstrated a negative correlation of the proportion of skilled health personnel attended deliveries with the MMR, stillbirth rate and neonatal mortality rate.

CONCLUSION: According to the national data of the included African countries, skilled delivery attendance was associated with significant reduction of maternal, fetal and neonatal mortality. SSA countries need to benchmark the experience of the North African countries to reduce the high maternal and perinatal deaths.
\end{abstract}

KEY WORDS: Africa, maternal mortality, perinatal mortality, skilled person attended delivery, systematic review

DOI: http://dx.doi.org/10.4314/ejhs.v24i1.7S

\section{INTRODUCTION}

Maternal Mortality Ratio (MMR), perinatal mortality rate (PMR) and the proportion of births attended by skilled health personnel are used as indicators for the status of maternal and perinatal health at national or regional level $(1,2)$. The 2013 World Health Statistics report has shown that the mean MMRs estimated for low and high income countries for the year 2010 were 410 and 14 per 100,000 live births, respectively (3). Similarly, according to World Health Organization (WHO) estimation, the PMR in high income countries was more than six times less than that of the low income countries (4).

\footnotetext{
${ }^{1}$ Hawassa University, College of Medicine and Health Sciences, Department of Gynecology-Obstetrics

${ }^{2}$ Hawassa University, College of Medicine and Health Sciences, Department of Pharmacology

Corresponding Author: Yifru Berhan, Email: yifrub@yahoo.com
} 
A joint statement by WHO, International Federation of Gynecology and Obstetrics (FIGO) and the International Confederation of Midwives advocates for skilled care for all women during pregnancy, childbirth and the immediate postnatal period (4). Furthermore, one of the reasons why the developed nations achieved a very low MMR and PMR is because they have already achieved hundred percent antenatal care, skilled health personnel attended deliveries and postpartum care $(5,6)$. In low income countries, however, high perinatal mortality has been attributed to several skilled person unattended deliveries $(5,7,8)$. The implication is, among others, the high maternal and perinatal deaths in low income countries were likely linked to extremely low utilization of skilled birth attendants $(6,9)$.

This happened despite the known fact that the majority of maternal and perinatal deaths due to obstetric complications are preventable provided that optimal care is delivered to the pregnant women and their babies during pregnancy, delivery and after birth (6, 10). Specifically, increasing the skilled health personnel attended deliveries has been recognized since long ago as a crucial intervention to reduce maternal and perinatal mortalities due to unbooked emergencies occurring during delivery and in the early postpartum period (11-13).

As a result, "zero tolerance for maternal deaths", primarily by increasing the rate of health service utilization by pregnant women, is the motto currently spoken out in every corner (14). This ambition comes primarily from examples of the developed countries' achievement. Furthermore, the absence of single maternal death in some health facilities among middle income countries with relatively high skilled health personnel attended deliveries was a stimulus to strive for maternal mortality reduction. For instance, it was reported that no maternal death occurred out of 49,239 pregnant women who were attended over two years in the Achimota Hospital in Ghana (15).

In the majority of African countries, however, it was estimated that about half of the births took place at home without the assistance of skilled health personnel (16). Several demographic and health surveys (17) have also shown a high prevalence of home delivery in Africa, but little is known how strongly are skilled person unattended deliveries are associated with maternal and perinatal mortality. Therefore, the aim of this review is to assess the gross correlation of MMRs and PMRs with the proportion of skilled health personnel attended deliveries in 41 African countries.

\section{METHODS}

\section{Search strategy}

In this study, systematic review was conducted. A computerize based literature search from the accessible electronic databases (MEASURE DHS, PUBMED, MEDLINE, Google Scholar, BIOINFO, HINARI, Chocrane Library and WHO) were performed by two investigators (YB and $A B)$. The search strategy was run with the specified database from 1990 through September 2013.

\section{Inclusion criteria and study selection}

For this systematic review, 41 African countries' national representative survey data on MMR, stillbirth rate, the neonatal mortality rate and the proportion of skilled health personnel attended deliveries were included. African countries with no report for these four variables were excluded from analysis.

\section{Operational definition}

Since all the included surveys used the same instrument developed by MEASURE DHS (17), terms which are the interest of this study (maternal mortality ratio, rates of skilled person attended delivery, cesarean delivery, stillbirth and neonatal mortality) were defined similarly. Maternal mortality ratio was defined as total number of maternal deaths per 100,000 live births. As WHO defined, skilled health personnel attended delivery is a care provided by "an accredited health professional such as a midwife, doctor or nursewho has been educated and trained to proficiency in the skills needed to manage normal (uncomplicated) pregnancies, childbirth and the immediate postnatal period, and in the identification, management and referral of complications in women and newborns" $(18,19)$. Caesarean delivery rate was defined as the total number abdominal route of delivery after 28 
weeks of gestation per 1000 total births. Stillbirth rate and neonatal mortality rates were defined as total number of fetal deaths per 1000 total births and neonatal deaths per 1000 live births in the first week of life after 28 weeks of gestation, respectively.

\section{Statistical analysis}

Bivariate regression analysis was done for the proportion of skilled person attended deliveries or cesarean deliveries in the $\mathrm{Y}$-axis and MMR or stillbirth rates or neonatal mortality rates in the $\mathrm{X}$ axis. Specifically, linear regression analyses were done for MMR, stillbirth rate and neonatal mortality rate in relation to skilled person attended delivery. Logarithmic regression analysis was done to assess the association of MMR with stillbirth rate. The association of MMR with caesarean delivery rate was assessed by exponential regression. Bivariate Pearson correlation coefficients ( $r$ ) were determined using SPSS software.

\section{RESULTS}

\section{Brief description of the methodological quality of the included studies}

DHS data were collected in developing countries using similar instrument for over twenty years. The included surveys data were cross sectional by design, nationally representative and large sample size. Almost all the surveys followed a standard procedure of data collection and data presentation. In the majority of the surveys, a two-stage cluster sampling design and strata for urban and rural households were used to select study respondents. The estimations for WHO data were made based on evidences available in 2012 using standardized categories and methods in order to enhance cross national comparability (3). It was also pointed out that these estimates were subject to considerable uncertainty, especially for countries low statistical and health and health information systems.

\section{Findings of the review}

Thirty-seven of the 41 DHS included in this review were from Sub Saharan African countries. Figure 1 shows the proportion of skilled health personnel attended deliveries and MMRs of 41 African countries at the national level as estimated by WHO (3) and MEASURE DHS (17). Among included countries, 27 have already achieved more than 50\% skilled health personnel attended deliveries. The highest proportion of health facility deliveries (74\%-100\%) was actually reported from all North African, some Southern and West African countries. African countries with relatively small population size (0.5-10 million) and middle to high income (3) were found to have above $90 \%$ skilled health personnel attended deliveries: Mauritious (100\%), Botswana (99\%), Libya (98\%), Tunisia and Algeria (each 95\%), and Congo (94\%). The proportion of skilled health personnel attended deliveries in Somalia, Ethiopia, Chad, and Niger were extremely low, i.e. less than $20 \%$.

With few exceptions (Congo, Malawi, Zimbabwe, Cameroon, Lesotho, Sierra Leone and Burundi), where both the proportion of skilled health personnel attended deliveries and MMRs were high, the majority of the included African countries with a high proportion of skilled health personnel attended deliveries (60\%-100\%) were able to reduce the maternal mortality to the range of 56-370 per 100,000 live births. Seven African countries have already achieved an MMR of less than 100 per 100,000 live births. Interestingly, some African countries (Uganda, Gambia, Ghana, Togo and Kenya), with $<60 \%$ skilled person attended deliveries, were able to reduce the MMR to $<=370$ per 100,000 live births. It was also noted that although the proportion of skilled person attended deliveries were extremely low in Ethiopia and Niger, the MMRs were in the lower high range. 

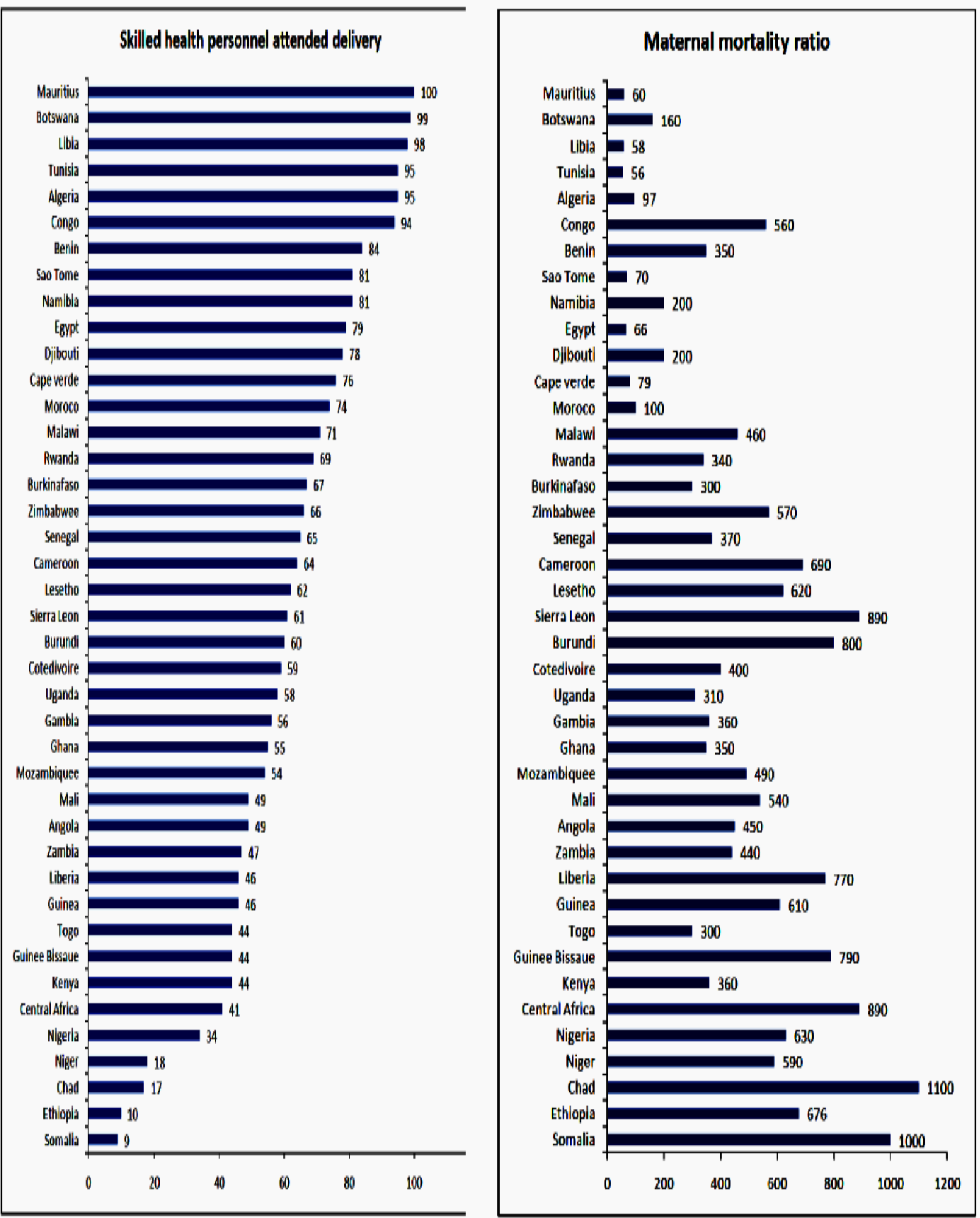

Congo $=$ Republic of Congo or Congo Brazzaville

Figure 1: The comparison of proportion of skilled health personnel attended delivery with maternal mortality ratio per 100,000 live births in 41 African countries $(2005-2012)$ 
In the regression analysis, as shown in Figure 2, it was observed that the proportion of skilled health personnel attended deliveries at the national level had a negative correlation with the national MMR $(\mathrm{r}=-0.7 ; \mathrm{P}<0.0001)$. The regression line demonstrated that the majority of African countries with a high proportion of skilled health personnel attended deliveries were having low MMR. In other words, the MMRs of most of the Sub Saharan Africa (SSA) countries with large population size (>10 million) were above the regression line.

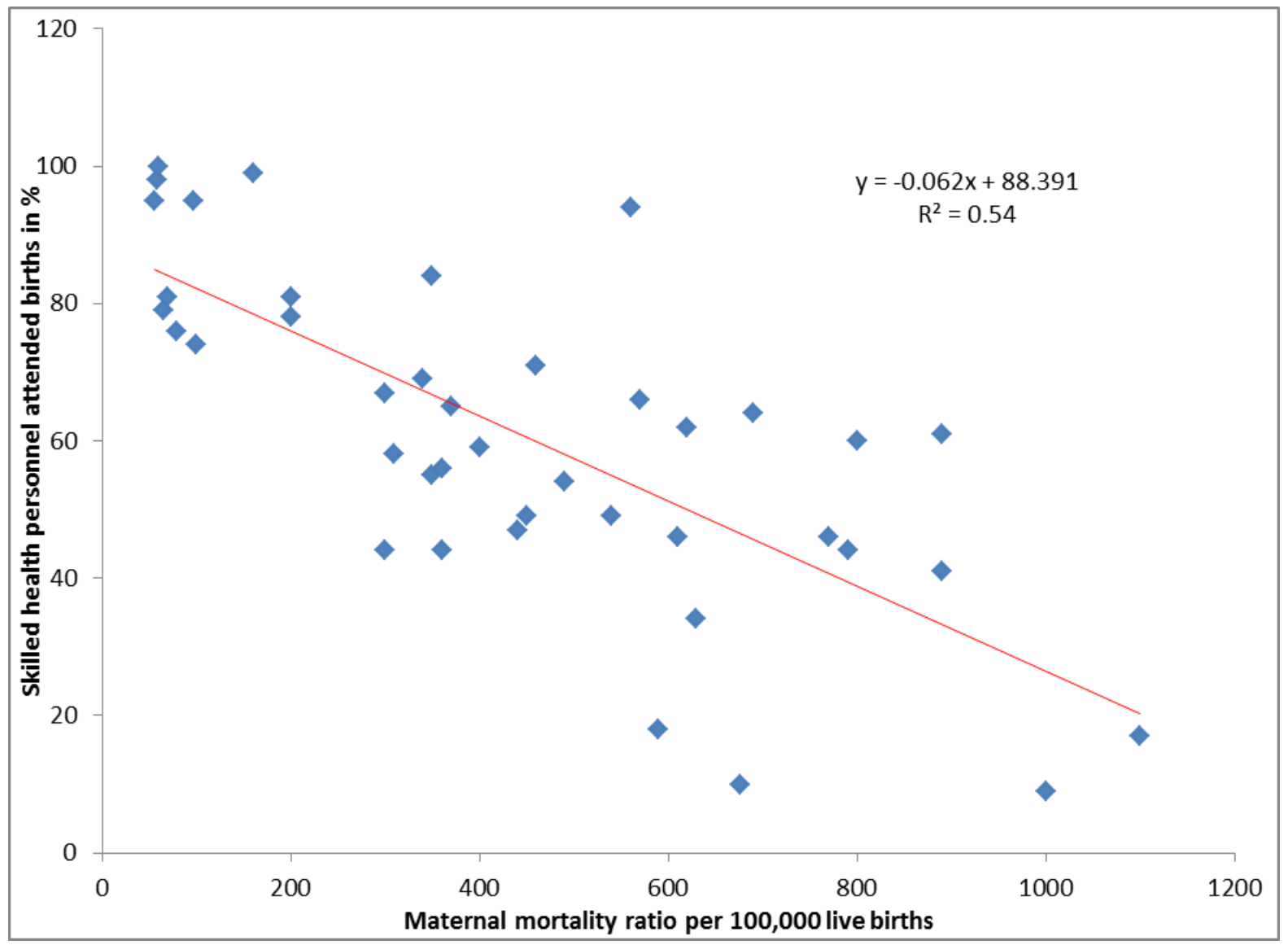

Figure 2: Linear regression analysis of maternal mortality ratio with skilled health personnel attended delivery using national survey data of 41 African countries. Pearson correlation coefficient $(r)=-0.7 ; P<$ 0.0001

The national estimated stillbirth rate was also inversely related to the proportion of skilled person attended deliveries $(\mathrm{r}=-0.6 ; \mathrm{P}<0.0001)$ (Figure 3). There are, however, a few exceptions (Djibuti, Senegal, Sierra Leone) where the stillbirth rates were among the highest (> 30 per 1000 total births) while the proportion of skilled person attended deliveries was above $60 \%$. In general, the top six countries with a high stillbirth rate were Nigeria (44/1000 total births), Djibuti and Senegal (each 34/1000 total births), Sierra Leone, Somalia and Guinee Bissaue (each 30/1000 total births). Among the best achievers, the top six countries with a stillbirth rate in the range of 9-15 per 1000 total births were Egypt, Algeria, Tunisia, Libya, Namibia and Mauritius. 


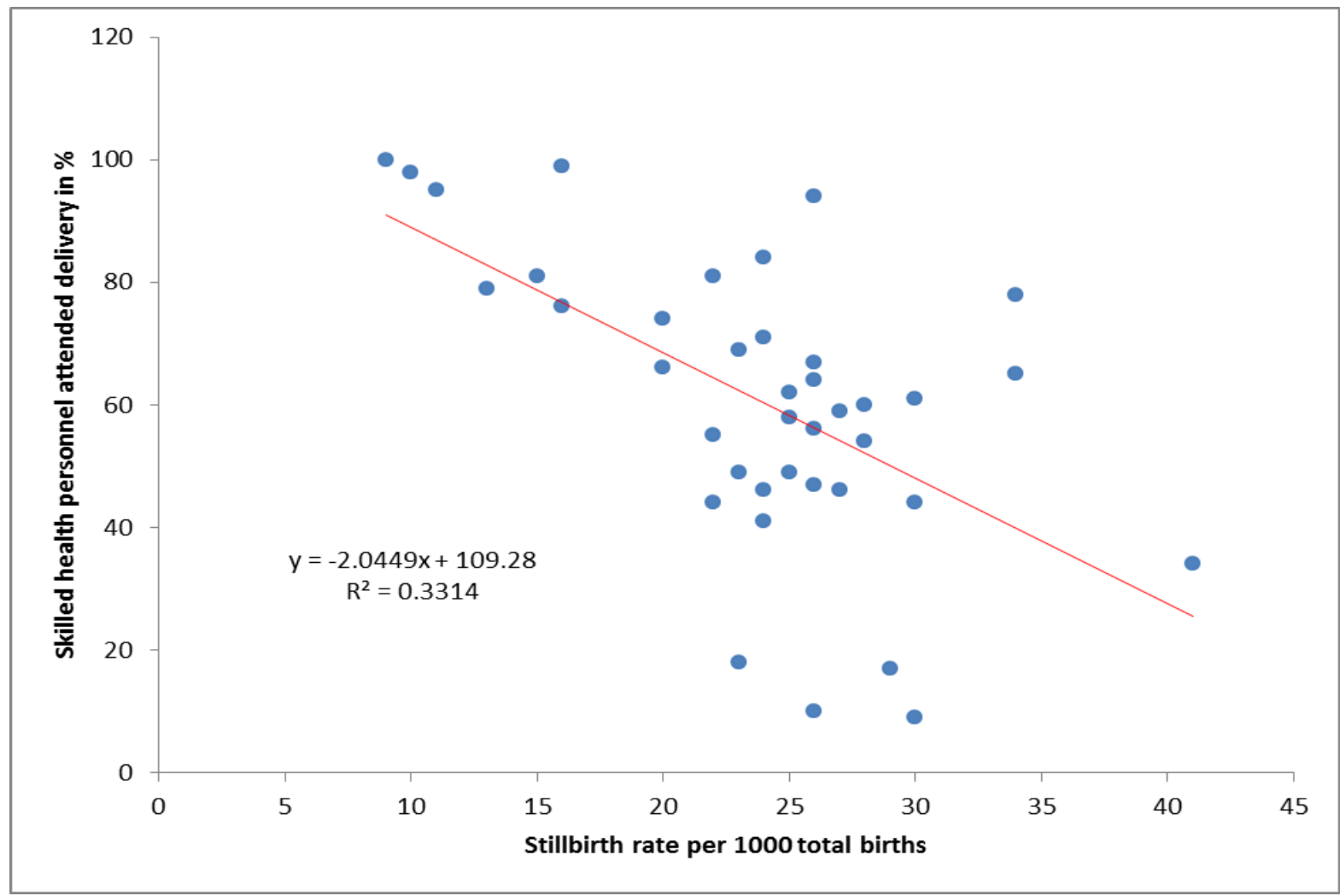

Figure 3: Linear regression analysis of stillbirth rate with skilled health personnel attended delivery using national survey data of 41 African countries. Pearson correlation coefficient $(r)=-0.6 ; P<0.0001$

Figure 4 shows how high stillbirths were complementarily occurring with high maternal mortalities. The regression line demonstrated a positive correlation of MMR with the stillbirth rate $(\mathrm{r}=0.6 ; \mathrm{P}<0.0001)$. A similar picture is observed in the regression analysis of MMR with neonatal mortality rate $(\mathrm{r}=0.8 ; \mathrm{P}<0.0001)$. This is to mean that African countries with high MMR had also high stillbirth and high neonatal mortality rates, which is a consolidating evidence for the findings indicated in Figures 2 and 3. 


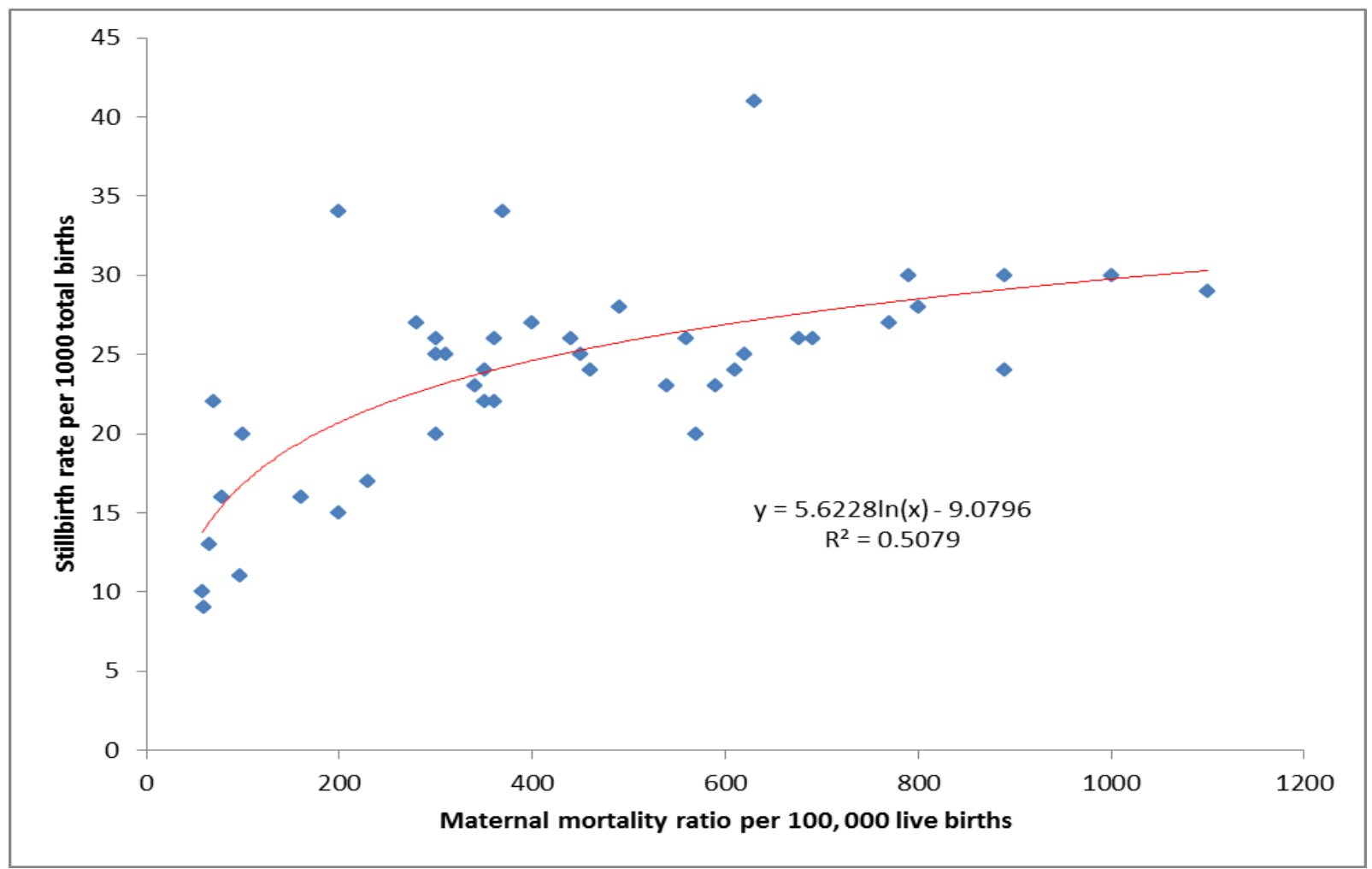

Figure 4: Logarithmic regression analysis of maternal mortality ratio with stillbirth rate using national survey data of 41 African countries; Pearson correlation coefficient $(r)=0.6 ; P<0.0001$

As shown in Figure 5, the regression analysis of the national neonatal mortality rate with the proportion of skilled person attended deliveries has also shown a similar pattern (as shown in Figure 3). It needs to be noted that some African countries have already reduced the neonatal mortality rate to 10 or less per 1000 live births
(Egypt, Tunisia, Libya, Cape Verde and Mauritius). The highest neonatal mortality rates (40-50 neonatal deaths per 1000 live births) were reported from Somalia, Sierra Leone, Mali, Burundi, Central Africa, Chad, CoteDivore, Guine Bissau and Angola. 


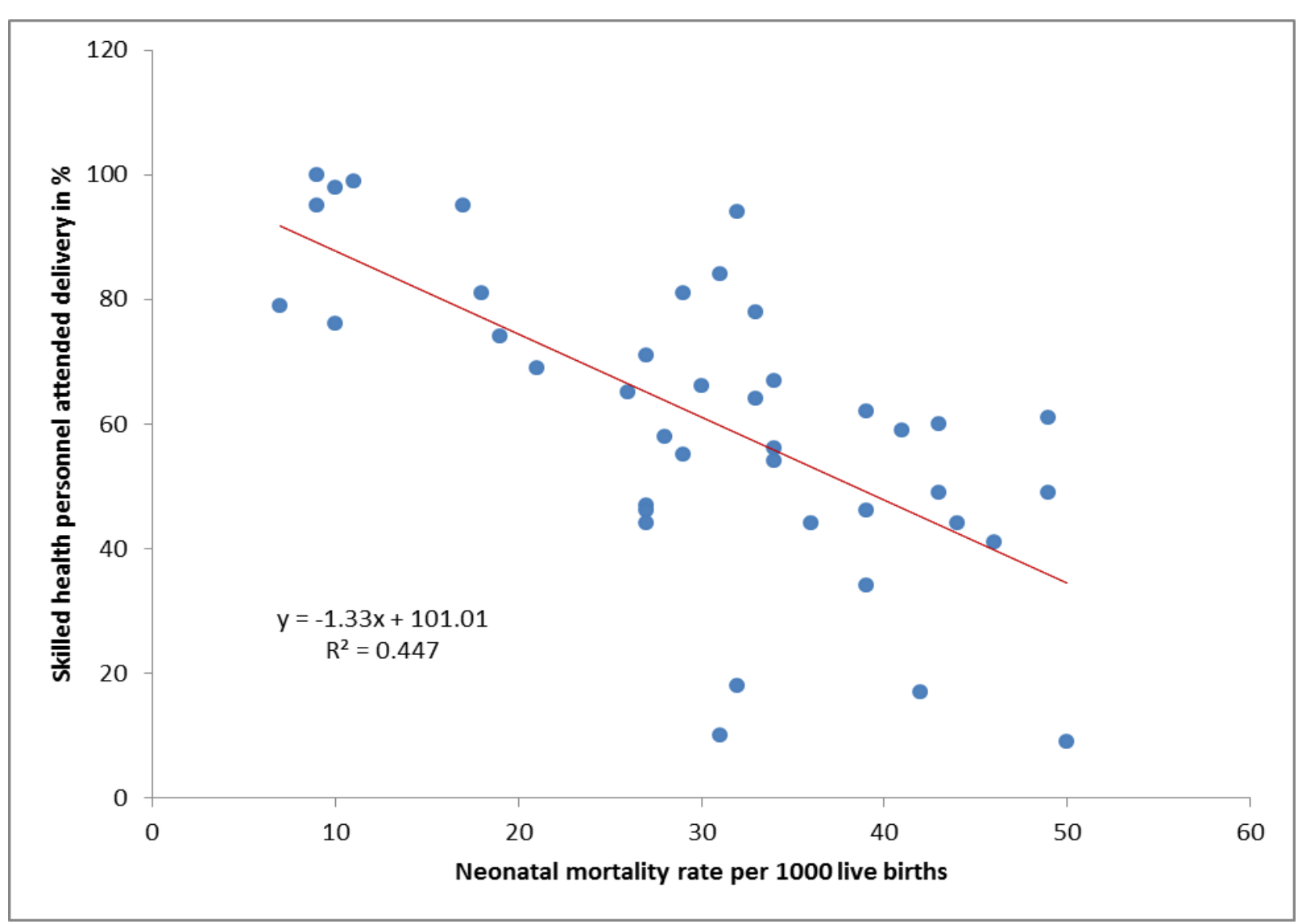

Figure 5: Linear regression analysis of neonatal mortality rate with skilled health personnel attended delivery using national survey data of 41 African countries. Pearson correlation coefficient $(r)=-0.6 ; P<$ 0.0001

The finding in Figure 6 is also another consolidating finding for the observed negative correlation of maternal deaths, stillbirths and neonatal deaths with a skilled person attended deliveries (as shown in Figures 2, 3, 5). Among 30 African countries, which reported the cesarean delivery rate and MMR, it was observed that an increase in cesarean delivery rate (probably indirectly an increase in proportion of skilled person attended deliveries) was correlated with a reduction in MMR $(r=-0.59 ; \mathrm{P}<0.0001)$. Similar regression lines were observed in the correlation analyses of stillbirth and neonatal mortality rates with the rate of cesarean delivery $(r=-0.6 ; \mathrm{P}<$ 0.0001 and $\mathrm{r}=-0.7 ; \mathrm{P}<0.0001$, respectively). Countries with $<=2 \%$ national cesarean deliveries include Ethiopia, Guinea, Mali, Niger, Chad, Nigeria and Burkina Faso. 


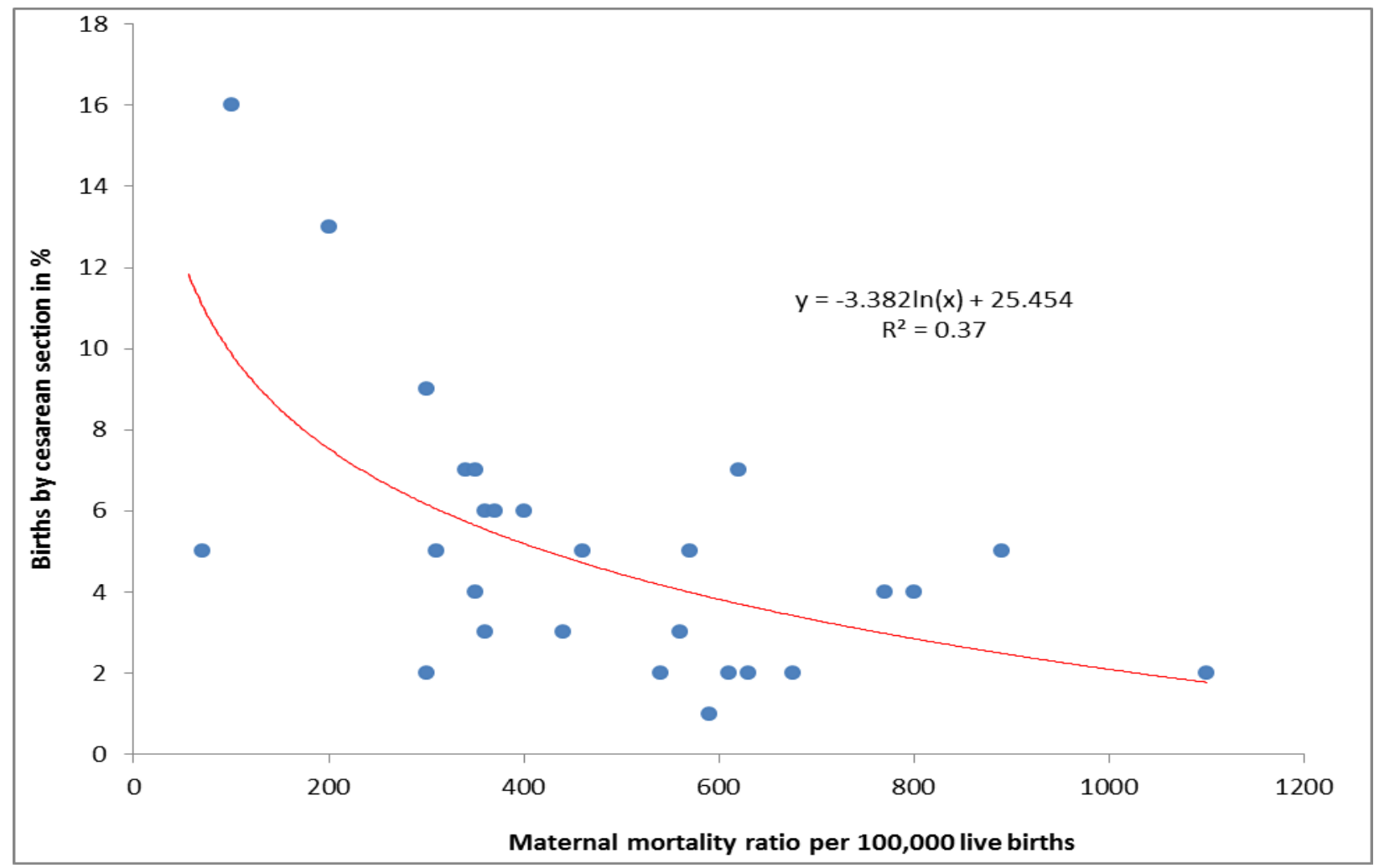

Figure 6: Exponential regression analysis of maternal mortality ratio with cesarean delivery using national survey data of 30 African countries. Pearson correlation coefficient $(r)=-0.59 ; P<0.0001$

\section{DISCUSSION}

This review has demonstrated the negative correlation of percentage of skilled health personnel attended delivery with MMR, stillbirth rate and neonatal mortality rate, which is consistent with previous reports $(20,21)$. In other words, included African countries with a low proportion of skilled people attended delivery had high MMR, high stillbirth rate and high neonatal mortality rate.

The progressively increasing maternal and perinatal deaths with the progressively declining proportion of skilled person attended delivery may show how significantly determinant the skilled person care during delivery is. In low income countries, however, it is known that multiple clinical care unrelated factors (education, income, culture, religion, geographic inaccessibility, lack of transport, and others) also contribute to the high maternal and perinatal mortality $(19,22-24)$. As far as clinical care for pregnant women is concerned, a significant reduction in maternal and perinatal mortality is possible when it is commenced early in pregnancy and continued to puerperium (25), which might be the case in some African countries which were able to reduce the maternal and perinatal deaths close to the level of some developed nations (3).

In other words, the negative correlation of MMR and stillbirth rate with the proportion of skilled person attended delivery can be taken as one of the proxy indicators for the status of obstetric care provided in the included African countries. The positive correlation of MMR with stillbirth rate was another evidence how maternal mortality was also accompanied by perinatal mortality in settings where the practice of skilled person attended delivery were very low. Another review has also shown that the majority of maternal and neonatal deaths occur in regions where most births occur without a skilled person attended delivery (26).

However, the finding of increased PMR among women with skilled person unattended delivery should be interpreted very cautiously. This is because several other exposure variables like delay in arrival to skilled person care and 
'push to hospital' obstetric complications and well known risk factors for perinatal deaths were not controlled. Specifically, the common factors for increased risk of PMR (hypertensive disorders, antepartum hemorrhage, preterm labor, premature rupture of fetal membranes, multiple pregnancy and obstructed labor with or without uterine rupture) (27-30) were not included in this review.

A similar finding in Indonesia was explained by the fact that women with low uptake of health professional birth attendance seek health care when they were ill and might be too late to save their lives (30). Otherwise, there are a lot of evidences that demonstrated the significant reduction in maternal and perinatal mortality among women who were under the care of skilled health professionals during childbirth $(5-9,31$, 32).

In this review, the proportion of cesarean delivery was taken as a proxy indicator of modern health care utilization by pregnant women during labor and delivery in the included African countries. Taking into account the inevitable obstetric and medical indications, WHO recommends $5 \%-15 \%$ cesarean delivery for the general population (33). Less than 5\% cesarean section indicates that there is unmet need; as a result, several mothers and babies are dying or complicating.

Lack of caesarean delivery service is known to increase the risk of maternal and fetal complications due to obstructed labor (34). In subSaharan Africa, vesicovaginal fistulas due to obstructed labor are highly prevalent (between 30,000 and 130,000 new cases occurring each year) (35). Another good example is the incidence of obstetric fistula in Ethiopia where the proportions of the skilled person attended delivery and cesarean delivery between 1995 and 2011 at the national level were about $5 \%-10 \%$ and $<1 \%$, respectively (36). As a result, in this country, it was reported that more than 26,000 fistula patients were not yet repaired in 2008 (37) although the country have five hospitals only for fistula repair.

The point is, the high proportion of obstetric fistula in some Sub Saharan African countries like Sudan, Ethiopia, Chad, Ghana, and Nigeria (38) is likely the reflection of the low skilled health personnel attended delivery including caesarean section. In other words, had these and other similar cases been under the care of skilled person during labor and delivery and offered timely cesarean section, there would not have been single case of obstetric fistula; and the observed high maternal and perinatal mortality could have been reduced.

In general, although there may be several other factors, the significant drop of maternal and perinatal deaths in North Africa seems to be linked with a significant increase in skilled person attended births. In 1990, the proportion of deliveries with a skilled attendant in North Africa and SSA were $40 \%$ and $41 \%$, respectively (39). In 2003, in North Africa, the proportion of skilled person attended delivery increased to $76 \%$ while the SSA remained same (41\%) (39). This is one of the reasons why in contemporary obstetrics, skilled health personnel attended delivery is highly recommended to reduce maternal and perinatal mortality to the lowest level $(4,40)$.

This study is not without limitations. Being a gross estimation of the correlation of the national MMR, stillbirth rate and neonatal death rate with the national proportion of skilled health personnel, delivery may not represent specific areas within the country.

In conclusion, the majority of SSA countries had high maternal deaths, high stillbirths and high neonatal deaths and all were negatively correlated with the proportion of skilled person attended delivery. Although such gross correlation cannot be definitely taken as a sole determinant factor, the consistency of the inverse relationship between skilled person attended delivery (including cesarean section) and maternal/perinatal deaths was a supportive evidence for the high maternal and perinatal deaths in SSA, where the skilled birth attendance utilization was very low. About two decades ago, the proportion of skilled person attended deliveries in SSA and North Africa was the same (each about 40\%). Therefore, the SSA countries need to benchmark the experience (about 90\% skilled birth attendance) of the North African countries to reduce the maternal and perinatal deaths.

\section{ACKNOWLEDGEMENT}

We would like to thank PANE and KMG Ethiopia for the modest financial support granted for this review. We are also grateful to UNFPA Ethiopia 
for their generous financial support to cover the publication fee.

\section{REFERENCES}

1. United Nations: The Millennium Development Goals report: Statistical annex. New York; 2007. Last accessed, Sept 2013: http://www.un.org/millenniumgoals.

2. Safe Motherhood Newsletter: Making pregnancy safer: a strategy for action SMN. 2002, 29.2. Last accessed, Sept 2013: https://apps.who.int/rht/msm/safe_motherhood _newsletter

3. World health organization. World Health Statistics 2013. Last accessed, Sept 2013: http://www.who.int/gho/publications/world_h ealth_statistics/EN_WHS2013/pdf

4. World Health Organization. Making pregnancy safer: the critical role of skilled attendants. Last accessed, Sept 2013: www.who.int/making_pregnancy_safer/docu ments/9241591692/en

5. World Health Organization. Neonatal and perinatal mortality: country, regional \& global estimates. Geneva, Switzerland, 2006. Last accessed, Sept 2013: whqlibdoc.who.int/publications/2006/9241563 206_eng.pdf

6. World Health Organization. Maternal mortality. Fact sheet number 348. 2012. Last accessed, Sept 2013: http://www.who.int/mediacentre/factsheets/fs3 48/en/

7. Feresu SA, Harlow SD, Welch K, Gillespie $\mathrm{BW}$. Incidence of stillbirth and perinatal mortality and their associated factors among women delivering at Harare Maternity Hospital, Zimbabwe: a cross-sectional retrospective analysis. BMC Pregnancy and Childbirth 2005, 5:9. http://www.biomedcentral.com

8. Lawn J, Cousens S, Zupan J. 4 million deaths. When? Where? Why? Lancet 2005; 365: 891900.

9. Kruk ME, Paczkowski MM, Tegegn A, et al. Women's preferences for obstetric care in rura 1 Ethiopia: a population-based discrete choice experiment in a region with low rates of facility delivery. J Epidemiol Community Health. 2010; 64(11):984-8.
10. Center for Diseases Control and Prevention. Morbidity and Mortality Weekly Report. 2001. Atlanta, U.S.A. Last accessed, Sept 2013: http://www.cdc.gov

11. Walraven GE, Mkanje RJ, van Roosmalen J, van Dongen PW, Dolmans WM. Perinatal mortality in home births in rural Tanzania. Eur J Obstet Gynecol Reprod Biol 1995; 58(2):131-4.

12. Howlader AA, Kabir M, Bhuiyan MM. Health seeking behavior of mothers and factors affecting infant and child mortality. Demography India 1999; 28(2):225-238.

13. World Health Organization: Reconciling maternal, newborn and child health with health system development. Chapter 7 in World Health Report 'Make every mother and child count' Geneva, Switzerland: WHO; 2005.

14. AFRICAN Medical and Research Foundation (AMREF). Zero tolerance for maternal death. Last accessed, Sept 2013: http://allafrica.com/stories/201307040064.htm

15. Aglobitse MK. Ghanaian Times. Achimota Hosp. Records Zero Maternal Deaths. Last accessed, Sept 2013: http://www.myradiogoldlive.com.

16. World Health Organization: Proportion of births attended by a skilled health worker. Geneva, Switzerland: WHO; 2008. Last accessed, sept 2013: http://www.who.int

17. Publication search by country (DHS): Last accessed, September 2013: http://www.measuredhs.com/pubs/

18. Safe Motherhood: Providing Emergency Obstetric and Neonatal Care to All in Need. Last accessed, Sept 2013: http://www.unfpa.org/public/home/mothers/

19. WHO, ICM, FIGO: Making pregnancy safer: the critical role of the skilled attendant. Geneva: World Health Organization; WHO DoRHaR 2004.

20. Moyer CA, Dako-Gyeke P, Adanu RM. Facility-based delivery and maternal and early neonatal mortality in sub-Saharan Africa: A regional review of the literature. Afr J Reprod Health 2013; 17(3): 30-43

21. Weiner R, Ronsmans C, Dorman E, Jilo $\mathrm{H}$, Muhoro A, Shulman C. Labor complications remain the most important risk factors for perinatal mortality in rural Kenya. 
Bull World Health Organ 2003; 81(8):561-6. Epub 2003 Oct 14

22. Getachew B, Yifru B. Perinatal mortality and associated risk factors: a case control study. Ethiopian J Health Scie 2012; 22(3): 153-162.

23. Bellad MB, Srividhya K, Ranjit K, Dhaded SM. Factors Associated with Perinatal Mortality: A Descriptive Observational Study. South Asian Fed Obstet Gynecol 2010; 2(1):49-51.

24. Schmiegelow C, Minja D, Oesterholt M, et al. Factors associated with and causes of perinatal mortality in Northeastern Tanzania. Acta Obstet Gynecol Scand 2012; 91(9):1061-8.

25. Ronsmans C, Scott S, Qomariyah SN, et al. Maternal mortality and professionally-assisted births in Indonesia. Bull World Health Organ 2009; 87: 416-423

26. Tumwine JK, Dungare PS. Maternity waiting shelters and pregnancy outcome: experience from a rural area in Zimbabwe. Ann Trop Paediatr 1996; 16(1):55-9.

27. Chandramohan D, Cutts F, Millard P. The effect of stay in a maternity waiting home on perinatal mortality in rural Zimbabwe. $J$ Trop Med Hyg 1995; 98(4):261-7.

28. Rahman S, Salameh K, Bener A, Ansari EL. Socioeconomic associations of improved maternal, neonatal, and perinatal survival in Qatar. Int J Wom Health 2010, 2:311-318.

29. Surekha AT, Kumar N. Socio demographic factors affecting perinatal mortality- A study in a rural setup. IOSR Journal of Pharmacy 2012; 2(4): 43-46).

30. Lawn JE, Cousens S, Zupan J. 4 million deaths: When? Where? Why? The Lancet 2005; 365 (9462):891 - 900.

31. Gunnar Kvåle G, Olsen BE, Hinderaker SG, Ulstein M, Bergsjø P. Maternal deaths in developing countries: A preventable tragedy. Norsk Epidemiologi 2005; 15 (2): 141-149.

32. Darmstadt GL, Lee ACC, Cousens S, et al. 60 million non-facility births: Who can deliver in community settings to reduce intrapartumrelated deaths? Int J Gynaecol Obstet 2009; 107(Suppl 1): S89-112.

33. World Health Organization. Appropriate technology for birth. Lancet 1985; 2: 436437.
34. Wall LL. Obstetric vesicovaginal fistula as an international public-health problem. Lancet 2006; 368(9542):1201

35. Vangeenderhuysen C, Prual A, Ould el Joud D. Obstetric fistulae: incidence estimates for sub-Saharan Africa. Int J Gynaecol Obstet 2001; 73(1):65-66.

36. Central Statistical Agency [Ethiopia] and ICF International. 2012. Ethiopia Demographic and Health Survey 2011. Addis Ababa, Ethiopia and Calverton, Maryland, USA: Central Statistical Agency and ICF International.

37. Wakabi W. Ethiopia steps up fight against fistula. The Lancet 2008; 371:1493-1494.

38. United Nations Population Fund and EngenderHealth. Obstetric fistula needs assessment report: Findings from nine African countries. New York (NY): United Nations Population Fund and Engender Health, 2003. Last accessed, Sept 2013: http://www.unfpa.org/fistula/docs/fistulaneeds-assessment.pdf.

39. World Health Organization. Progress towards the Millennium Development Goals, 19902005. Last accessed, Sept 2013: http://unstats.un.org/unsd/mi/goals

40. Buor D, Bream K. An analysis of the determinants of maternal mortality in subSaharan Africa. $J$ Womens Health (Larchmt) 2004; 13(8):926-38. 